\title{
Intellektuelles Kapital messen, bewerten und steuern
}

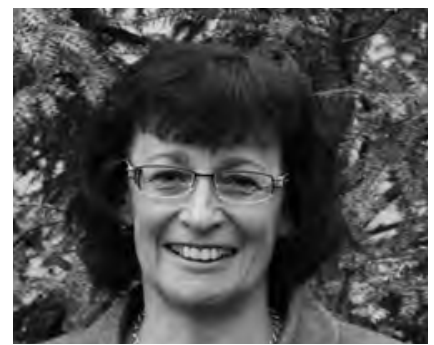

VON SUSANNE KIRCHHOFFKESTEL

Prof. Dr. habil. Susanne KirchhoffKestel war als wissenschaftliche Assistentin an der Wirtschaftsund Sozialwissenschaftlichen Fakultät der TU Dortmund tätig und forschte dort zur Thematik der Non-Profit-Organisationen. Seit Februar 2010 hat sie eine Professur für Betriebswirtschaftslehre, Management Sozialer Dienstleistungen an der Universität Vechta inne.

E-Mail susanne.kirchhoff-kestel@ uni-vechta.de

\author{
Der Wert eines Unternehmens besteht nicht nur aus \\ den buchhalterisch erfassbaren Größen. Insbesondere \\ in sozialen Dienstleistungsorganisationen stellt \\ das Intellektuelle Kapital der Mitarbeitenden \\ oft den entscheidenden Faktor für die \\ Qualität der Leistungserstellung dar.
}

Steigender Wettbewerbsdruck für soziale Organisationen, zunehmende Qualitätsanfordernisse bei der Erstellung von sozialen Dienstleistungen, aber auch Veränderungen und Zunahme des Bedarfs kennzeichnen die Entwicklung der Rahmenbedingungen, unter denen prozess ein. An den Nutzern wird die Leistung erbracht, sie tragen aber auch selbst zur Art und Qualität der Leistungserstellung bei. Zudem können die Leistungsempfänger die Qualität der vom Leistungsanbieter erbrachten Leistung häufig im vorhinein nur schwer

\section{"strukturkapital ist das Wissen, das abends nicht nach Hause geht"}

soziale Organisationen arbeiten. Damit steigt die Relevanz von erfolgsversprechenden Strategien, um sich im "Markt « der sozialen Dienstleistungen behaupten zu können. Soziale Organisationen sind besonders auf ihre immateriellen Ressourcen, wie die Motivation und Qualifikation der Mitarbeiter, eine gute Kommunikationsstruktur, ein vertrauenswürdiges Image, angewiesen, wenn sie sich »strategische Wettbewerbsvorteile« sichern wollen.

Die Leistungserstellung in sozialen Organisationen lässt sich dadurch charakterisieren, dass größtenteils immaterielle Güter, wie Pflege, Beratung und Betreuung erstellt werden und der Prozess der Leistungserstellung durch eine hohe Personalintensität gekennzeichnet ist. Die Leistungsempfänger greifen als externer Faktor in den Dienstleistungs- beurteilen, so dass eine gute Reputation für soziale Organisationen von besonderer Bedeutung ist (vgl. Bono 2006, S. 29-32). Immaterielle Ressourcen haben somit eine hohe Relevanz für die Leistungserstellung sozialer Organisationen.

Vertreter des Intellectual CapitalAnsatzes stellen die immateriellen Ressourcen, wie die Fähigkeiten und Kompetenzen der Mitarbeitenden, gute organisationale Strukturen sowie Kundenzufriedenheit und Reputation in den Mittelpunkt ihrer Betrachtungen und sehen diese als besonders relevante Einflussfaktoren auf den Erfolg der Unternehmung an (vgl. z. B. Edvinson, Brünig 2000, S. 11-13 und 17 f.; Stewart 1998, S. 66-73).

Kong und Thomsen, zwei australische Forscher, haben ermittelt, dass 
Intellektuelles Kapital auch für NonProfit-Organisationen ein wichtiges Konzept ist. Mittels Literaturanalyse und qualitativer Interviews fanden sie bezogen auf soziale Non-Profit-Organisationen heraus, dass die Bewertung des Intellektuellen Kapitals

- das Verständnis dafür erhöht, wie immaterielle Ressourcen für die Langzeitentwicklung genutzt werden können, und somit eine wichtige Basis für die strategische Planung darstellt.

- dazu führt, sich mit der eigenen Identität zu beschäftigen. Dabei geht es um die Fragen, welche Fähigkeiten und Wissen in der eigenen Organisation vorhanden sind und wohin man sich entwickeln will. Dies führt zu einer Fokussierung auf die Mission und die sozialen Ziele.

- als gemeinsame Sprache dienen kann, um organisationale Fähigkeiten und nicht monetäre Leistungen wichtigen Stakeholdern (z. B. Sponsoren, Banken) zu kommunizieren (vgl. Kong, Thomsen 2006, S. 218-227; Kong 2003, S. 470 f.).

Intellektuelles Kapital wird oft in $\mathrm{Hu}-$ mankapital, Strukturkapital und Beziehungskapital klassifiziert:

- Humankapital wird charakterisiert als »das, was denkt" (Renzl et al. 2006, S. 235). Damit sind alle individuellen Fähigkeiten, Erfahrungen und die Motivation der Mitarbeitenden gemeint.

- Strukturkapital ist »jenes Wissen, das abends nicht nach Hause geht" (Renzl et al. 2006, S. 235), wie beispielsweise Datenbanken, Patente, Strategie und Kultur, Strukturen und Informationssysteme sowie Abläufe. Strukturkapital benötigen die Mitarbeiter, um produktiv und innovativ agieren zu können.

- Beziehungskapital stellt die Beziehungen zu Kunden, Partnern, Kapitalgebern etc. und die Netzwerke mit Dritten dar. Der Wert dieses Kapitals wird beispielsweise sichtbar in Kundenzufriedenheit oder Reputation (vgl. Stewart 1998, S. 84-86; Edvinson, Brüning 2000, S. 28-32; Arbeitskreis Wissensbilanz et al. 2008, S. 18-20).

\begin{tabular}{|c|c|}
\hline $\begin{array}{l}\text { Intellektuelles } \\
\text { Kapital }\end{array}$ & $\begin{array}{l}\text { Exemplarische Indikatoren für soziale Organisationen } \\
\text { (Beispiele) }\end{array}$ \\
\hline Humankapital & $\begin{array}{l}\text { Zahl der haupt- und ehrenamtlich Mitarbeitenden } \\
\text { Zahl der voll- und teilzeitbeschäftigt Mitarbeitenden } \\
\text { O-ualifikation, Berufserfahrung der Mitarbeitenden, Dauer } \\
\text { der Zugehörigkeit zur Organisation } \\
\text { - Kompetenzen (z. B. Fachkompetenz, Führungskompetenz, so- } \\
\text { ziale Kompetenz) } \\
\text { Zufriedenheit, Motivation der Mitarbeitenden } \\
\text { christliche Werteorientierung der Mitarbeitenden } \\
\text { Weiterbildungstage pro Mitarbeitendem, Supervisionen }\end{array}$ \\
\hline Strukturkapital & $\begin{array}{l}\text { Vorhandensein eines Qualitätsmanagementsystems } \\
\text { vorhandensein einer IT-Struktur, Investitionen in IT-Struktur } \\
\text { a Vorhandensein einer Unternehmenskultur }\end{array}$ \\
\hline Beziehungskapital & $\begin{array}{l}\text { Ausgaben für Öffentlichkeitsarbeit } \\
\text { - Anzahl öffentlichkeitswirksamer Events } \\
\text { - Anzahl der Spender, Förderer und Mitglieder } \\
\text { - Anzahl der Kooperationen und Know-how-Partnerschaften } \\
\text { glaubwürdiges Image, Reputation }\end{array}$ \\
\hline
\end{tabular}

Abb. 1: Das Intellektuelle Kapital einer Organisation lässt sich anhand zahlreicher Indikatoren umfassend beschreiben.

Diese Bestandteile des Intellektuellen Kapitals lassen sich auch auf soziale Organisationen übertragen (vgl. Abb. 1). Die Menge dieser Indikatoren zeigt bereits, dass jede soziale Organisation für sich festlegen muss, welche Elemente des Intellektuellen Kapitals für sie von Bedeutung sind, um Wettbewerbsvorteile zu erreichen. Ebenso ist zwischen Indikatoren zu unterscheiden, die den Input charakterisieren und Indikatoren, die den Prozess und die Leistungsergebnisse (Output) sowie die Leistungswirkungen (Outcome) beschreiben. Die ganzheitliche Messung, Bewertung und Steuerung des Intellektuellen Kapitals kann durch eine Wissensbilanz vorgenommen werden (vgl. Abb. 2).

Ausgehend von vorgegebenen und selbst definierten Zielen und Strategien geht das Intellektuelle Kapital als Input in die Erstellung der sozialen Dienstleistung ein. Die Leistungsprozesse - hier beispielhaft dargestellt an den Kernprozessen der eigentlichen sozialen Dienstleistung, internen Dienstleistungen (z. B. Personalverwaltung, Buchhaltung) und Networking, Öffentlichkeitsarbeit und Fundraising - führen zu Leistungsergebnissen und zu Wirkungen auf die Stakeholder. Beispielsweise können die Qualifikation und Motivation der Mitarbeitenden in der Altenpflege über qualitätsmäßig gute Pflegeprozesse sich positiv auf die Zufriedenheit der Bewohner und die Reputation des Pflegeheims auswirken.

Für die Anwendung in der Praxis ist die Ermittlung, Bewertung und Steuerung des Intellektuellen Kapitals als Teilaspekt der strategischen Planung und Steuerung anzusehen. Die strategi-

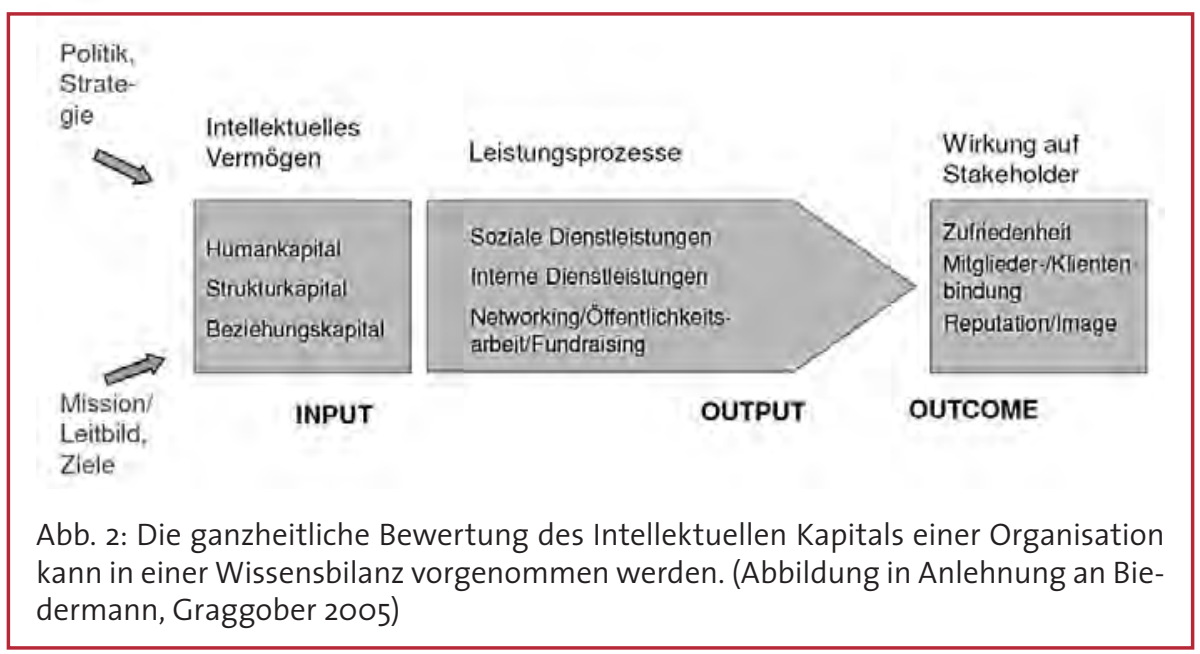




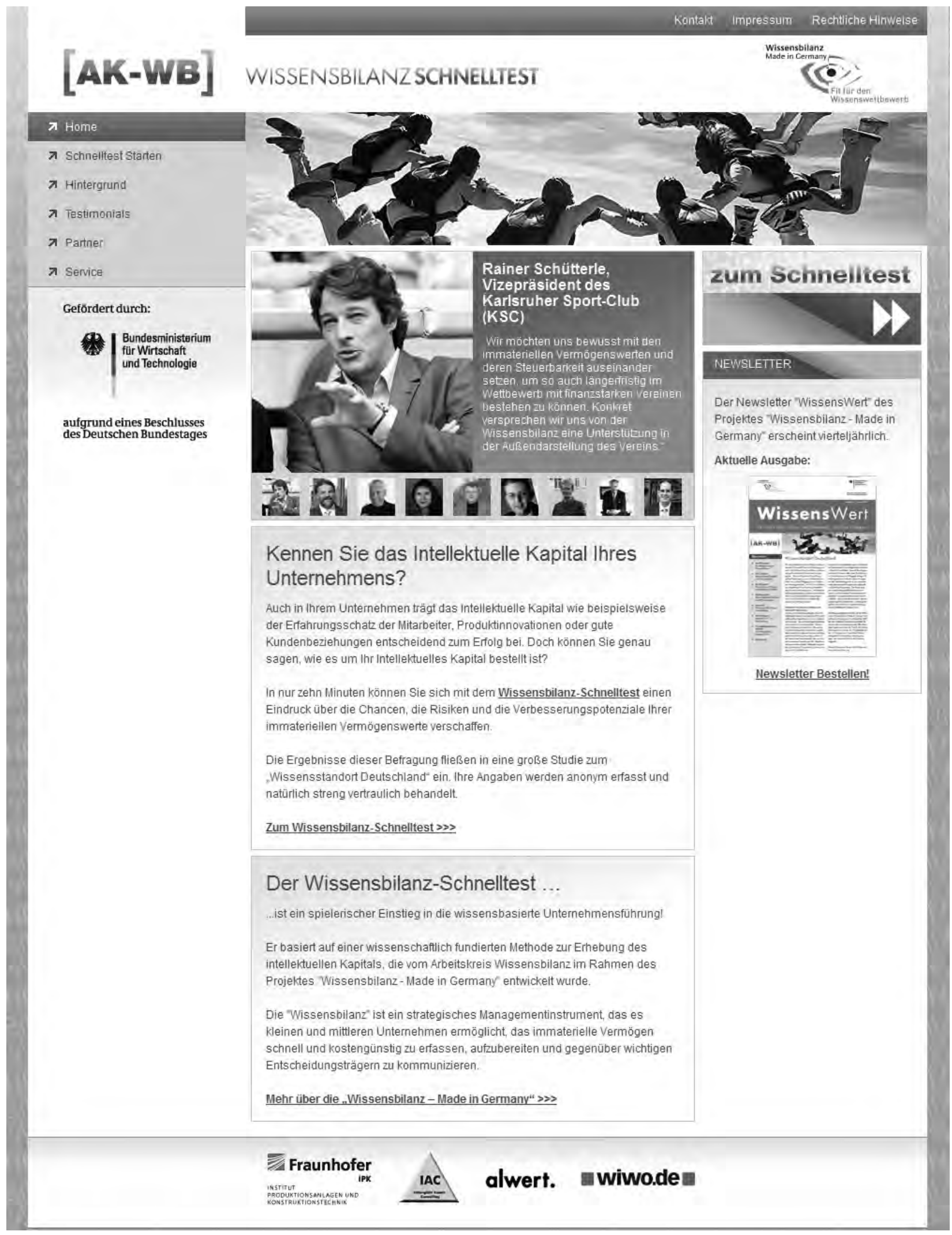

Die Wissensbilanz ist ein strategisches Managementinstrument, das es kleinen und mittleren Unternehmen ermöglicht, das immaterielle Vermögen schnell und kostengünstig zu erfassen, aufzubereiten und gegenüber wichtigen Entscheidungsträgern darzustellen. Die Website »Wissensbilanz-Schnelltest« bietet dazu einen Fragenkatalog, mit dem sich Unternehmen in zehn Minuten einen ersten Eindruck ihrer immateriellen Vermögenswerte verschaffen können. Der Test basiert auf einer Methode zur Erhebung des Intellektuellen Kapitals, die vom Arbeitskreis Wissensbilanz im Rahmen des Projektes "Wissensbilanz - Made in Germany" entwickelt wurde. Internet http://www.wissensbilanz-schnelltest.de 
sche Ausrichtung der Organisation hat zwar Einfluss auf die Bewertung und Steuerung des Intellektuellen Kapitals, andererseits kann es durch die Erstellung einer Wissensbilanz wiederum zu einer Anpassung der Strategie kommen (vgl. Arbeitskreis Wissensbilanz et al. 2008, S. 45).

Angestoßen durch ein vom Bundesministerium für Wirtschaft und Arbeit finanziertes Pilotprojekt im Jahr 2004 haben auch einige soziale Organisationen in Deutschland mit der Erstellung von Wissensbilanzen begonnen: die Bundeszentrale des Deutschen Caritasverbandes in Freiburg, der Caritasverband Leipzig, eine Caritas Fortbildungsakademie, die Diakonie Rosenheim,

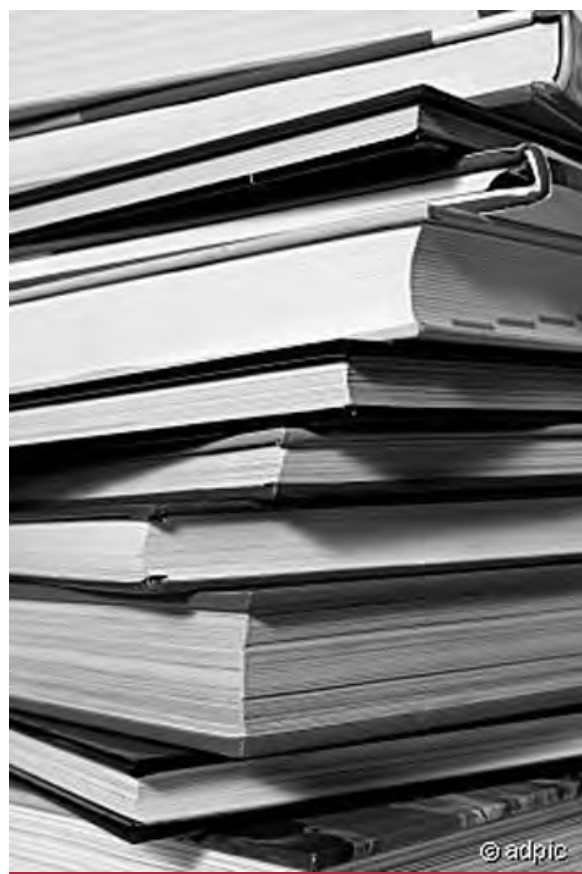

\section{Literatur}

Arbeitskreis Wissensbilanz, Alwert, K.; Bornemann, W. Fraunhofer Institut für Produktionsanlagen und Konstruktionstechnik (2008): Wissensbilanz. Made in Germany. Leitfaden 2.0 zur Erstellung einer Wissensbilanz. Hg.: Bundesministerium für Wirtschaft und Technologie, Dokumentation Nr. 574, Berlin. Internet http:// www.akwissensbilanz.org/Infoservice/Infomaterial/WB-Leitfaden_2.o.pdf.

\section{Arbeitskreis Wissensbilanz (2011): Wissens-}

Wert. Aktuelles Rund um die »Wissensbilanz -

Made in Germany". Februar 2011. S. 3.

Biedermann, H.; Graggober, M. (2005): Die Wissensbilanz als Strategie- und Steuerungsinstrument im Forschungsmanagement. In: eine Fachklinik und der Deutsche Entwicklungsdienst.

Diese Organisationen sehen die Wissensbilanz als wichtiges Instrument der Unternehmensführung an, das hilft die Wirkungszusammenhänge der Einflussfaktoren auf den Erfolg zu ermitteln, neue Kooperationspartner und Finanzgeber zu gewinnen und sich gut in das Qualitätsmanagement und das Rechnungswesen integrieren lässt (vgl. z. B. Winterhalter 2005; Arbeitskreis Wissensbilanz 2011, S. 3.)

Diakonie und Caritas benennen in ihren gemeinsam entwickelten Transparenzstandards zudem die Wissensbilanz als »Kann-Modul« für den Geschäftsbericht (vgl. Diakonie, Caritas 2010).

Mertins, K., Alwert, K.; Heisig, P. (Hg.): Wissensbilanzen. Intellektuelles Kapital erfolgreich nutzen und entwickeln. Berlin Heidelberg New York, S. 187-202.

Bono, M. L. (2006): NPO Controlling. Professionelle Steuerung sozialer Dienstleistungen, Stuttgart.

Diakonisches Werk der evangelischen Kirche in Deutschland, Deutscher Cariatasverband (Hg.) (2010): Transparenzstandards für Caritas und Diakonie. Internet http://www.caritas. de/75205.html.

Edvinson, L.; Brünig, G. (2000): Aktivposten Wissenskapital. Unsichtbare Werte bilanzierbar machen. Wiesbaden.

Kong, E. (2003) Using Intellectual Capital as a Strategic Tool for Non-profit Organisations. In: The International Journal of Knowledge, Culture und Change Management, Vol. 3, p. 467-474. Kong, E.; Thomsen, S. B. (2006) Intellectual capital and strategic human resource management in social service non-profit organisations in Australia. In: International Journal of Human Resources Development and Management, Vol. 6, No. 2/3/4, p. 213-231.

Renzl, B.; Matzler, K.; Huemer, S.; Rothenberger, S. (2006): Wissensbilanzierung an Universitäten. In: Matzler, K.; Hinterhuber, $\mathrm{H}$. H.; Renzl, B.; Rothenberger, S. (Hg.): Immaterielle Vermögenswerte. Handbuch der intangible Assets. Berlin. S. 231-249.

Stewart, T.A. (1998): Der vierte Produktionsfaktor. Wachstum und Wettbewerbsvorteile durch Wissensmanagement. München, Wien.

Winterhalter, B. (2005) Wissensbilanzen.

Besseres Ranking für soziale Organisationen. In: SOZIALwirtschaft, Nr. 2, S. 6-8.

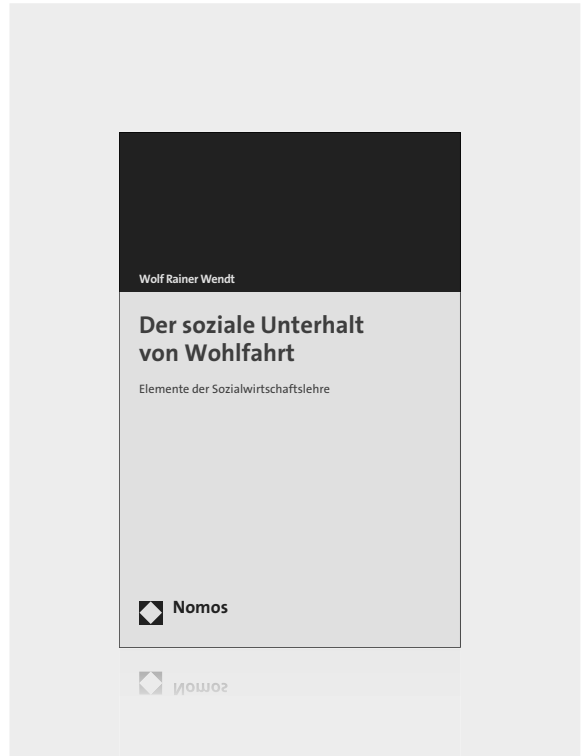

\section{Der soziale Unterhalt von Wohlfahrt}

Elemente der Sozialwirtschaftslehre Von Wolf Rainer Wendt

2011, 178 S., brosch., 39,-€

ISBN 978-3-8329-7000-0

Sozialwirtschaft umfasst im System der sozialen Sicherung und Versorgung die Vielfalt der Unternehmungen und Leistungen zur individuellen und gemeinschaftliche Wohlfahrt. Für ihren Unterhalt stehen im Sozialund Gesundheitswesen die Organisationen, Einrichtungen und Dienste der öffentlichen und sozialen Daseinsvorsorge ein.

Die Pflege von Wohlfahrt in „Diensten am Menschen“ ist produktiv. Ihre Ökonomie im Haushalt des Sozialstaats und in Beziehung auf die Lebensführung im Haushalt von Personen und in Gemeinschaft ist Gegenstand dieses Buches.

www.nomos-shop.de/14092

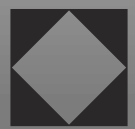

Nomos 